\title{
Construction of Frames for Shift-Invariant Spaces
}

\author{
Stevan Pilipović ${ }^{1}$ and Suzana Simić $^{2}$ \\ ${ }^{1}$ Department of Mathematics and Informatics, Faculty of Science, University of Novi Sad, \\ Trg Dositeja Obradovica 4, 21000 Novi Sad, Serbia \\ ${ }^{2}$ Department of Mathematics and Informatics, Faculty of Science, University of Kragujevac, Radoja Domanovića 12, \\ 34000 Kragujevac, Serbia \\ Correspondence should be addressed to Suzana Simić; suzanasimic@kg.ac.rs
}

Received 9 May 2013; Accepted 25 July 2013

Academic Editor: Satit Saejung

Copyright @ 2013 S. Pilipović and S. Simić. This is an open access article distributed under the Creative Commons Attribution License, which permits unrestricted use, distribution, and reproduction in any medium, provided the original work is properly cited.

We construct a sequence $\left\{\phi_{i}(\cdot-j) \mid j \in \mathbb{Z}, i=1, \ldots, r\right\}$ which constitutes a $p$-frame for the weighted shift-invariant space $V_{\mu}^{p}(\Phi)=$ $\left\{\sum_{i=1}^{r} \sum_{j \in \mathbb{Z}} c_{i}(j) \phi_{i}(\cdot-j) \mid\left\{c_{i}(j)\right\}_{j \in \mathbb{Z}} \in \ell_{\mu}^{p}, i=1, \ldots, r\right\}, p \in[1, \infty]$, and generates a closed shift-invariant subspace of $L_{\mu}^{p}(\mathbb{R})$. The first construction is obtained by choosing functions $\phi_{i}, i=1, \ldots, r$, with compactly supported Fourier transforms $\widehat{\phi}_{i}, i=1, \ldots, r$. The second construction, with compactly supported $\phi_{i}, i=1, \ldots, r$, gives the Riesz basis.

\section{Introduction and Preliminaries}

The shift-invariant spaces $V_{\mu}^{p}(\Phi), p \in[1, \infty]$, quoted in the abstract, are used in the wavelet analysis, approximation theory, sampling theory, and so forth. They have been extensively studied by many authors [1-18]. The aim of this paper is to construct $V_{\mu}^{p}(\Phi), p \in[1, \infty]$, spaces with specially chosen functions $\phi_{i}, i=1, \ldots, r$, which generate its $p$-frame. These results extend and correct the construction obtained in [19]. For the first construction, we take functions $\phi_{i}, i=1, \ldots, r$, so that the Fourier transforms are compactly supported smooth functions. Also, we derive conditions for the collection $\left\{\phi_{i}(\cdot-\right.$ j) $\mid j \in \mathbb{Z}, i=1, \ldots, r\}$ to form a Riesz basis for $V_{\mu}^{p}(\Phi)$. We note that the properties of the constructed frame guarantee the feasibility of a stable and continuous reconstruction algorithm in $V_{\mu}^{p}(\Phi)[20]$. We generalize these results for a shift-invariant subspace of $L_{\mu}^{p}\left(\mathbb{R}^{d}\right)$. The second construction is obtained by choosing compactly supported functions $\phi_{i}$, $i=1, \ldots, r$. In this way, we obtain the Riesz basis.

This paper is organized as follows. In Section 2 we quote some basic properties of certain subspaces of the weighted $L^{p}$ and $\ell^{p}$ spaces. In Section 3 we derive conditions for functions of the form $\widehat{\phi}_{i}(\cdot)=\theta\left(\cdot+k_{i} \pi\right), k_{i} \in \mathbb{Z}, i=1,2, \ldots, r, r \in \mathbb{N}$, to form a Riesz basis for $V_{\mu}^{p}(\Phi)$. We also show that using functions of the form $\widehat{\phi}_{i}(\cdot)=\theta(\cdot+i \pi), i=1, \ldots, r$, where $\theta$ is compactly supported smooth function whose length of support is less than or equal to $2 \pi$, we cannot construct a $p$-frame for the shift-invariant space $V_{\mu}^{p}(\Phi)$. In Section 4 we construct a sequence $\left\{\phi_{i}(\cdot-j) \mid j \in \mathbb{Z}, i=0, \ldots, r\right\}$, where $r \in$ $2 \mathbb{N}$ or $r \in 3 \mathbb{N}$, which constitutes a $p$-frame for the weighted shift-invariant space $V_{\mu}^{p}(\Phi)$. Our construction shows that the sampling and reconstruction problem in the shift-invariant spaces is robust in the sense of [1]. In Section 5 we construct $p$-Riesz basis by using compactly supported functions $\phi_{i}, i=$ $1, \ldots, r$.

\section{Basic Spaces}

Let a function $\omega$ be nonnegative, continuous, symmetric, and submultiplicative; that is, $\omega(x+y) \leq \omega(x) \omega(y), x, y \in \mathbb{R}^{d}$; let a function $\mu$ be $\omega$-moderate; that is, $\mu(x+y) \leq C \omega(x) \mu(y)$, $x, y \in \mathbb{R}^{d}$. Functions $\mu$ and $\omega$ are called weights. We consider the weighted function spaces $L_{\mu}^{p}$ and the weighted sequence 
spaces $\ell_{\mu}^{p}\left(\mathbb{Z}^{d}\right)$ with $\omega$-moderate weights $\mu$ (see [19]). Let $p \in$ $[1, \infty)$. Then (with obvious modification for $p=\infty$ )

$$
\begin{aligned}
& \mathscr{L}_{\omega}^{p}=\left\{f \mid\|f\|_{\mathscr{L}_{\omega}^{p}}\right. \\
& =\left(\int_{[0,1]^{d}}\left(\sum_{j \in \mathbb{Z}^{d}}|f(x+j)| \omega(x+j)\right)^{p} d x\right)^{1 / p} \\
& <+\infty\} \text {, } \\
& W_{\omega}^{p}:=\left\{f \mid\|f\|_{W_{\omega}^{p}}\right. \\
& \left.=\left(\sum_{j \in \mathbb{Z}^{d}} \sup _{x \in[0,1]^{d}}|f(x+j)|^{p} \omega(j)^{p}\right)^{1 / p}<+\infty\right\} .
\end{aligned}
$$

In what follows, we use the notation $\Phi=\left(\phi_{1}, \ldots, \phi_{r}\right)^{T}$. Define $\|\Phi\|_{\mathscr{H}}=\sum_{i=1}^{r}\left\|\phi_{i}\right\|_{\mathscr{H}}$, where $\mathscr{H}=L_{\omega}^{p}, \mathscr{L}_{\omega}^{p}$ or $W_{\omega}^{p}, p \in$ $[1, \infty]$. With $\mathscr{F} \phi=\widehat{\phi}$ we denote the Fourier transform of the function $\phi$; that is, $\widehat{\phi}(\xi)=\int_{\mathbb{R}^{d}} \phi(x) e^{-i \pi x \cdot \xi} \mathrm{d} x, \xi \in \mathbb{R}^{d}$.

Let $c=\left\{c_{i}\right\}_{i \in \mathbb{N}} \in \ell_{\mu}^{p}$ and $f, g \in L_{\omega}^{p}, p \in[1, \infty]$. We define, as in [1], the semiconvolution $f *^{\prime} c$ as $\left(f *^{\prime} c\right)(x)=$ $\sum_{j \in \mathbb{Z}^{d}} c_{j} f(x-j), x \in \mathbb{R}^{d}$, and $\langle f, g\rangle=\int_{\mathbb{R}^{d}} f(x) \overline{g(x)} \mathrm{d} x$.

The concept of a $p$-frame is introduced in [1].

It is said that a collection $\left\{\phi_{i}(\cdot-j) \mid j \in \mathbb{Z}^{d}, i=1, \ldots, r\right\}$ is a $p$-frame for $V_{\mu}^{p}(\Phi)$ if there exists a positive constant $C$ (dependent upon $\Phi$, $p$, and $\omega$ ) such that

$$
\begin{aligned}
& C^{-1}\|f\|_{L_{\mu}^{p}} \\
& \quad \leq \sum_{i=1}^{r}\left\|\left\{\int_{\mathbb{R}^{d}} f(x) \overline{\phi_{i}(x-j)} \mathrm{d} x\right\}_{j \in \mathbb{Z}^{d}}\right\|_{\ell_{\mu}^{p}} \\
& \quad \leq C\|f\|_{L_{\mu}^{p}, \quad f \in V_{\mu}^{p}(\Phi) .}
\end{aligned}
$$

Recall [21] that the shift-invariant spaces are defined by

$$
\begin{gathered}
V_{\mu}^{p}(\Phi):=\left\{f \in L_{\mu}^{p} \mid f(\cdot)=\sum_{i=1}^{r} \sum_{j \in \mathbb{Z}^{d}} c_{j}^{i} \phi_{i}(\cdot-j),\right. \\
\left.\left\{c_{j}^{i}\right\}_{j \in \mathbb{Z}^{d}} \in \ell_{\mu}^{p}, i=1, \ldots, r\right\} .
\end{gathered}
$$

Remark 1 (see [22]). Let $\Phi \in W_{\omega}^{1}$ and let $\mu$ be $\omega$-moderate. Then $V_{\mu}^{p}(\Phi)$ is a subspace (not necessarily closed) of $L_{\mu}^{p}$ and $W_{\mu}^{p}$ for any $p \in[1, \infty]$. Clearly (2) implies that $\ell_{\mu}^{p}$ and $V_{\mu}^{p}(\Phi)$ are isomorphic Banach spaces.

$$
\begin{aligned}
& \text { Let } \Phi=\left(\phi_{1}, \ldots, \phi_{r}\right)^{T} \text {. Let } \\
& {[\widehat{\Phi}, \widehat{\Phi}](\xi)=\left[\sum_{k \in \mathbb{Z}^{d}} \widehat{\phi}_{i}(\xi+2 k \pi) \overline{\widehat{\phi}_{j}(\xi+2 k \pi)}\right]_{1 \leq i \leq r, 1 \leq j \leq r},}
\end{aligned}
$$

where we assume that $\widehat{\phi}_{i}(\xi) \overline{\widehat{\phi}_{j}(\xi)}$ is integrable for any $1 \leq i$, $j \leq r$. Let $A=[a(j)]_{j \in \mathbb{Z}^{d}}$ be an $r \times \infty$ matrix and $A \overline{A^{T}}=$ $\left[\sum_{j \in \mathbb{Z}^{d}} a_{i}(j) \overline{a_{i^{\prime}}(j)}\right]_{1 \leq i, i^{\prime} \leq r}$. Then $\operatorname{rank} A=\operatorname{rank} A \overline{A^{T}}$.

We recall results from $[1,19]$ which are needed in the sequel.

Lemma 2 (see [1]). The following statements are equivalent.

(1) $\operatorname{rank}[\widehat{\Phi}(\xi+2 j \pi)]_{j \in \mathbb{Z}^{d}}$ is a constant function on $\mathbb{R}^{d}$.

(2) $\operatorname{rank}[\widehat{\Phi}, \widehat{\Phi}](\xi)$ is a constant function on $\mathbb{R}^{d}$.

(3) There exists a positive constant $C$ independent of $\xi$ such that

$$
\begin{aligned}
C^{-1}[\widehat{\Phi}, \widehat{\Phi}](\xi) & \leq[\widehat{\Phi}, \widehat{\Phi}](\xi) \overline{[\Phi, \widehat{\Phi}](\xi)^{T}} \\
& \leq C[\widehat{\Phi}, \widehat{\Phi}](\xi), \quad \xi \in[-\pi, \pi]^{d} .
\end{aligned}
$$

The next theorem [19] derives necessary and sufficient conditions for an indexed family $\left\{\phi_{i}(\cdot-j) \mid j \in \mathbb{Z}^{d}, i=\right.$ $1, \ldots, r\}$ to constitute a $p$-frame for $V_{\mu}^{p}(\Phi)$, which is equivalent with the closedness of this space in $L_{\mu}^{p}$. Thus, it is shown that under appropriate conditions on the frame vectors, there is an equivalence between the concept of $p$-frames, Banach frames, and the closedness of the space they generate.

Theorem 3 (see [19]). Let $\Phi=\left(\phi_{1}, \ldots, \phi_{r}\right)^{T} \in\left(W_{\omega}^{1}\right)^{r}, p_{0} \in$ $[1, \infty]$, and let $\mu$ be $\omega$-moderate. The following statements are equivalent.

(i) $V_{\mu}^{p_{0}}(\Phi)$ is closed in $L_{\mu}^{p_{0}}$.

(ii) $\left\{\phi_{i}(\cdot-j) \mid j \in \mathbb{Z}^{d}, i=1, \ldots, r\right\}$ is a $p_{0}$-frame for $V_{\mu}^{P_{0}}(\Phi)$.

(iii) There exists a positive constant $C$ such that

$$
\begin{aligned}
C^{-1}[\widehat{\Phi}, \widehat{\Phi}](\xi) & \leq[\widehat{\Phi}, \widehat{\Phi}](\xi) \overline{[\Phi, \widehat{\Phi}](\xi)^{T}} \\
& \leq C[\widehat{\Phi}, \widehat{\Phi}](\xi), \quad \xi \in[-\pi, \pi]^{d} .
\end{aligned}
$$

(iv) There exist positive constants $C_{1}$ and $C_{2}$ (depending on $\Phi$ and $\omega$ ) such that

$$
\begin{aligned}
C_{1}\|f\|_{L_{\mu}^{p_{0}}} & \leq \inf _{f=\sum_{i=1}^{r} \phi_{i} *^{\prime} c^{i}} \sum_{i=1}^{r}\left\|\left\{c_{j}^{i}\right\}_{j \in \mathbb{Z}^{d}}\right\|_{\ell_{\mu}^{p_{0}}} \\
& \leq C_{2}\|f\|_{L_{\mu}^{p_{0}}}, \quad f \in V_{\mu}^{p_{0}}(\Phi) .
\end{aligned}
$$


(v) There exists $\Psi=\left(\psi_{1}, \ldots, \psi_{r}\right)^{T} \in\left(W_{\omega}^{1}\right)^{r}$, such that

$$
\begin{aligned}
f & =\sum_{i=1}^{r} \sum_{j \in \mathbb{Z}^{d}}\left\langle f, \psi_{i}(\cdot-j)\right\rangle \phi_{i}(\cdot-j) \\
& =\sum_{i=1}^{r} \sum_{j \in \mathbb{Z}^{d}}\left\langle f, \phi_{i}(\cdot-j)\right\rangle \psi_{i}(\cdot-j), \quad f \in V_{\mu}^{p_{0}}(\Phi) .
\end{aligned}
$$

Corollary 4 (see [19]). Let $\Phi=\left(\phi_{1}, \ldots, \phi_{r}\right)^{T} \in\left(W_{\omega}^{1}\right)^{r}, p_{0} \in$ $[1, \infty]$, and let $\mu$ be $\omega$-moderate.

(i) If $\left\{\phi_{i}(\cdot-j) \mid j \in \mathbb{Z}^{d}, i=1, \ldots, r\right\}$ is a $p_{0}$-frame for $V_{\mu}^{p_{0}}(\Phi)$, then the collection $\left\{\phi_{i}(\cdot-j) \mid j \in \mathbb{Z}^{d}, i=\right.$ $1, \ldots, r\}$ is a $p$-frame for $V_{\mu}^{p}(\Phi)$ for any $p \in[1, \infty]$.

(ii) If $V_{\mu}^{p_{0}}(\Phi)$ is closed in $L_{\mu}^{p_{0}}$ and $W_{\mu}^{p_{0}}$, then $V_{\mu}^{p}(\Phi)$ is closed in $L_{\mu}^{p}$ and $W_{\mu}^{p}$ for any $p \in[1, \infty]$.

(iii) If (7) holds for $p_{0}$, then it holds for any $p \in[1, \infty]$.

\section{Construction of Frames Using a Band-Limited Function}

Considering the length of the support of a function $\theta$, we have different cases for the rank of matrix $[\widehat{\Phi}(\xi+2 j \pi)]_{j \in \mathbb{Z}}$.

First, we consider the next claim.

Let $\theta \in C_{0}^{\infty}(\mathbb{R})$ be a nonnegative function such that $\theta(x)>0, x \in(-\pi, \pi)$, and $\operatorname{supp} \theta \subseteq[-\pi, \pi]$. Moreover, let

$$
\widehat{\phi}_{k}(\xi)=\theta(\xi+k \pi), \quad k \in \mathbb{Z},
$$

and $\Phi=\left(\phi_{i}, \phi_{i+1}, \ldots, \phi_{i+r}\right)^{T}, i \in \mathbb{Z}, r \in \mathbb{N}$.

Then the rank of matrix $[\widehat{\Phi}(\xi+2 j \pi)]_{j \in \mathbb{Z}}$ is not a constant function on $\mathbb{R}$ and it depends on $\xi \in \mathbb{R}$.

As a matter of fact, by the Paley-Wiener theorem, $\phi_{i} \in$ $\delta(\mathbb{R}) \subset W_{\mu}^{1}(\mathbb{R}), i \in \mathbb{Z}$. For any $i \in \mathbb{Z}$, matrix $\left[\widehat{\phi}_{i}, \widehat{\phi}_{i}\right](\xi)=$ $\sum_{j \in \mathbb{Z}}|\theta(\xi+i \pi+2 j \pi)|^{2}, \xi \in \mathbb{R}$, has the rank 0 or 1 , depending on $\xi$. Moreover, we have $\left[\widehat{\phi}_{2 i}, \widehat{\phi}_{2 i}\right](\pi)=0$ and $\left[\widehat{\phi}_{2 i}, \widehat{\phi}_{2 i}\right](0)>0$. Because of that, the rank of the matrix $[\widehat{\Phi}(\xi+2 j \pi)]_{j \in \mathbb{Z}}$ is not a constant function on $\mathbb{R}$ and it depends on $\xi \in \mathbb{R}$.

Theorem 5. Let $\theta \in C_{0}^{\infty}(\mathbb{R})$ be a non-negative function such that $\theta(x)>0, x \in(-\pi-\varepsilon, \pi+\varepsilon)$, and $\operatorname{supp} \theta=[-\pi-\varepsilon, \pi+\varepsilon]$, where $0<\varepsilon<1 / 4$. Moreover, let

$$
\widehat{\phi}_{i}(\xi)=\theta\left(\xi+k_{i} \pi\right), \quad k_{i} \in \mathbb{Z}, i=1,2, \ldots, r,
$$

and $\Phi=\left(\phi_{1}, \phi_{2}, \ldots, \phi_{r}\right)^{T}$.

(1) If $\left|k_{2}-k_{1}\right|=2$ and $\left|k_{i}-k_{j}\right| \geq 2$ for different $i, j \leq r$, then

$$
\operatorname{rank}[\widehat{\Phi}(\xi+2 j \pi)]_{j \in \mathbb{Z}}=r, \quad \xi \in \mathbb{R} .
$$

(2) If $\left|k_{2}-k_{1}\right|=2$ and, at least for $k_{i_{1}}$ and $k_{i_{2}}$, it holds that $\left|k_{i_{1}}-k_{i_{2}}\right|=1$, where $1 \leq i_{1}, i_{2} \leq r$, then $\operatorname{rank}[\widehat{\Phi}(\xi+$ $2 j \pi)]_{j \in \mathbb{Z}}$ is not a constant function on $\mathbb{R}$.
Proof. By the Paley-Wiener theorem, $\phi_{i} \in \mathcal{S}(\mathbb{R}) \subset W_{\mu}^{1}(\mathbb{R})$, $i=1, \ldots, r$. All possible cases are described in the following lemmas.

Lemma 6. Let $\Phi=\left(\phi_{k_{1}}, \phi_{k_{2}}\right)^{T}, k_{2}-k_{1}=2, k_{1}, k_{2} \in \mathbb{Z}$. The rank of matrix $[\widehat{\Phi}(\xi+2 j \pi)]_{j \in \mathbb{Z}}$ is a constant function on $\mathbb{R}$ and equals 2.

Proof. We have the next two cases.

$\left(1^{\circ}\right)$ If $\xi \in\left(-\pi-\varepsilon-k_{1} \pi+2 \ell \pi,-\pi+\varepsilon-k_{1} \pi+2 \ell \pi\right), \ell \in \mathbb{Z}$, for matrix $[\widehat{\Phi}(\xi+2 j \pi)]_{j \in \mathbb{Z}}$ we obtain $2 \times \infty$ matrix

$$
\left[\begin{array}{ccccccc}
\cdots & 0 & 0 & a^{1} & b^{1} & 0 & \cdots \\
\cdots & 0 & a^{2} & b^{2} & 0 & 0 & \cdots
\end{array}\right]
$$

for some $a^{i}, b^{i}>0, i=1,2$. It is obvious that $\operatorname{rank}[\widehat{\Phi}(\xi+$ $2 j \pi)]_{j \in \mathbb{Z}}=2, \xi \in\left(-\pi-\varepsilon-k_{1} \pi+2 \ell \pi,-\pi+\varepsilon-k_{1} \pi+2 \ell \pi\right)$, $\ell \in \mathbb{Z}$.

$\left(2^{\circ}\right)$ For $\xi \in\left[-\pi+\varepsilon-k_{1} \pi+2 \ell \pi, \pi-\varepsilon-k_{1} \pi+2 \ell \pi\right]$, $\ell \in \mathbb{Z}$, there are only two nonzero values $a^{1}$ and $a^{2}$ which are in different columns of matrix $[\widehat{\Phi}(\xi+2 j \pi)]_{j \in \mathbb{Z}}$. Since

$$
[\widehat{\Phi}(\xi+2 j \pi)]_{j \in \mathbb{Z}}=\left[\begin{array}{cccccc}
\cdots & 0 & 0 & a^{1} & 0 & \cdots \\
\cdots & 0 & a^{2} & 0 & 0 & \cdots
\end{array}\right]_{2 \times \infty},
$$

it has the rank 2 for all $\xi \in\left[-\pi+\varepsilon-k_{1} \pi+2 \ell \pi, \pi-\varepsilon-k_{1} \pi+2 \ell \pi\right]$, $\ell \in \mathbb{Z}$.

We conclude that the rank of matrix $[\widehat{\Phi}(\xi+2 j \pi)]_{j \in \mathbb{Z}}, \Phi=$ $\left(\phi_{k_{1}}, \phi_{k_{2}}\right)^{T}, k_{2}-k_{1}=2, k_{1}, k_{2} \in \mathbb{Z}$, is a constant function on $\mathbb{R}$ and equals 2 .

Lemma 7. The rank of matrix $[\widehat{\Phi}(\xi+2 j \pi)]_{j \in \mathbb{Z}}$ is not a constant function on $\mathbb{R}$ if $\Phi=\left(\phi_{k_{1}}, \phi_{k_{2}}\right)^{T}, k_{2}-k_{1}=1, k_{1}, k_{2} \in \mathbb{Z}$.

Proof. We have four different cases for matrix $[\widehat{\Phi}(\xi+2 j \pi)]_{j \in \mathbb{Z}}$. Suppose, without losing generality, that $k_{1} \in 2 \mathbb{Z}$.

$\left(1^{\circ}\right)$ If $\xi \in(-\pi-\varepsilon+2 \ell \pi,-\pi+\varepsilon+2 \ell \pi), \ell \in \mathbb{Z}$, then

$$
\begin{array}{r}
{[\widehat{\Phi}(\xi+2 j \pi)]_{j \in \mathbb{Z}}=\left[\begin{array}{cccccc}
\cdots & 0 & a^{1} & b^{1} & 0 & \cdots \\
\cdots & 0 & a^{2} & 0 & 0 & \cdots
\end{array}\right],} \\
a^{1}, a^{2}, b^{1}>0,
\end{array}
$$

and $\operatorname{rank}[\widehat{\Phi}(\xi+2 j \pi)]_{j \in \mathbb{Z}}=2$, for all $\xi \in(-\pi-\varepsilon+2 \ell \pi,-\pi+$ $\varepsilon+2 \ell \pi), \ell \in \mathbb{Z}$.

$\left(2^{\circ}\right)$ For $\xi \in[-\pi+\varepsilon+2 \ell \pi,-\varepsilon+2 \ell \pi], \ell \in \mathbb{Z}$, nonzero values $a^{1}$ and $a^{2}$ are in the same column of matrix $[\widehat{\Phi}(\xi+2 j \pi)]_{j \in \mathbb{Z}}$. For any choice of a $2 \times 2$ matrix, we get that the determinant equals 0 . So we obtain

$$
\operatorname{rank}\left[\begin{array}{ccccc}
\cdots & 0 & a^{1} & 0 & \cdots \\
\cdots & 0 & a^{2} & 0 & \cdots
\end{array}\right]=1,
$$

for all $\xi \in[-\pi+\varepsilon+2 \ell \pi,-\varepsilon+2 \ell \pi], \ell \in \mathbb{Z}$.

$\left(3^{\circ}\right)$ If $\xi \in(-\varepsilon+2 \ell \pi, \varepsilon+2 \ell \pi), \ell \in \mathbb{Z}$, then

$$
[\widehat{\Phi}(\xi+2 j \pi)]_{j \in \mathbb{Z}}=\left[\begin{array}{cccccc}
\cdots & 0 & 0 & a^{1} & 0 & \cdots \\
\cdots & 0 & b^{2} & a^{2} & 0 & \cdots
\end{array}\right],
$$


for some $a^{1}, a^{2}, b^{2}>0$, has the rank 2 , for all $\xi \in(-\varepsilon+2 \ell \pi, \varepsilon+$ $2 \ell \pi), \ell \in \mathbb{Z}$.

$\left(4^{\circ}\right)$ For $\xi \in[\varepsilon+2 \ell \pi, \pi-\varepsilon+2 \ell \pi], \ell \in \mathbb{Z}$, there are two nonzero values $a^{1}$ and $b^{2}$ in different columns of matrix [ $\widehat{\Phi}(\xi+$ $2 j \pi)]_{j \in \mathbb{Z}}$ and the block with these elements determines the rank 2 for all $\xi \in[-\varepsilon+2 \ell \pi, \pi-\varepsilon+2 \ell \pi], \ell \in \mathbb{Z}$.

Considering possible cases, we conclude that $\operatorname{rank}[\widehat{\Phi}(\xi+$ $2 j \pi)]_{j \in \mathbb{Z}}, \Phi=\left(\phi_{k_{1}}, \phi_{k_{2}}\right)^{T}, k_{2}-k_{1}=1, k_{1}, k_{2} \in \mathbb{Z}$, depends on $\xi \in \mathbb{R}$ and equals 1 or 2 . This rank is a nonconstant function on $\mathbb{R}$.

Proof of Theorem 5. (1) Using Lemmas 6 and 7, it is obvious that if $\left|k_{2}-k_{1}\right|=2$ and $\left|k_{i}-k_{j}\right| \geq 2$ for different $i, j \leq r$, then the position of the first non-zero element in each row of matrix $[\widehat{\Phi}(\xi+2 j \pi)]_{j \in \mathbb{Z}}$ is unique for each row. So the rank of matrix $[\widehat{\Phi}(\xi+2 j \pi)]_{j \in \mathbb{Z}}$ is a constant function on $\mathbb{R}$ and equals $r$ for all $\xi \in \mathbb{R}$.

(2) If $\left|k_{2}-k_{1}\right|=2$ and, at least for $k_{i_{1}}$ and $k_{i_{2}}$, it holds that $\left|k_{i_{1}}-k_{i_{2}}\right|=1,1 \leq i_{1}, i_{2} \leq r$, then, in the row with the index $i_{2}$ (suppose, without losing generality, that $i_{2} \in 2 \mathbb{Z}+1$ ), we will have a new column with a non-zero element for $\xi \in(-\pi-\varepsilon+$ $2 \ell \pi,-\pi+\varepsilon+2 \ell \pi), \ell \in \mathbb{Z}$, but for $\xi \in[\varepsilon+2 \ell \pi, \pi-\varepsilon+2 \ell \pi]$, $\ell \in \mathbb{Z}$, the positions of all non-zero elements in that row will appear in the previous columns. It is obvious that the rank of the matrix $[\widehat{\Phi}(\xi+2 j \pi)]_{j \in \mathbb{Z}}$ depends on $\xi \in \mathbb{R}$ and is not the same for all $\xi \in \mathbb{R}$.

As a consequence of Theorems 3 and 5(1), we have the following result.

Theorem 8. Let the functions $\theta$ and $\Phi$ satisfy all the conditions of Theorem 5(1). Then space $V_{\mu}^{p}(\Phi)$ is closed in $L_{\mu}^{p}$ for any $p \in$ $[1, \infty]$, and the family $\left\{\phi_{i}(\cdot-j) \mid j \in \mathbb{Z}, 1 \leq i \leq r\right\}$ is a $p$-Riesz basis for $V_{\mu}^{p}(\Phi)$ for any $p \in[1, \infty]$.

The following theorem is a generalisation of Theorem 5 and can be proved in the same way, so we omit the proof.

Theorem 9. Let $\theta \in C_{0}^{\infty}(\mathbb{R})$ be a positive function such that $\theta(x)>0, x \in(a, b), b>a$, and supported by $[a, b]$ where $b-a>2 \pi$. Moreover, let

$$
\widehat{\phi}_{i}(\xi)=\theta\left(\xi+k_{i} \pi\right), \quad k_{i} \in \mathbb{Z}, i=1,2, \ldots, r, r \in \mathbb{N},
$$

and $\Phi=\left(\phi_{1}, \phi_{2}, \ldots, \phi_{r}\right)^{T}$.

(1) If $\left|k_{2}-k_{1}\right|=2$ and $\left|k_{i}-k_{j}\right| \geq 2$ for different $i, j \leq r$, then

$$
\operatorname{rank}[\widehat{\Phi}(\xi+2 j \pi)]_{j \in \mathbb{Z}}=r, \quad \xi \in \mathbb{R} .
$$

(2) If $\left|k_{2}-k_{1}\right|=2$ and, at least for $k_{i_{1}}$ and $k_{i_{2}}$, it holds that $\left|k_{i_{1}}-k_{i_{2}}\right|=1$, where $1 \leq i_{1}, i_{2} \leq r$, then $\operatorname{rank}[\widehat{\Phi}(\xi+$ $2 j \pi)]_{j \in \mathbb{Z}}$ is not a constant function on $\mathbb{R}$.

\section{Construction of Frames Using Several Band-Limited Functions}

Firstly, we consider two smooth functions with proper compact supports.
Lemma 10. Let $\theta \in C_{0}^{\infty}(\mathbb{R}), \psi \in C_{0}^{\infty}(\mathbb{R})$ be positive functions such that

$$
\begin{gathered}
\theta(x)>0, \quad x \in(-\varepsilon, 2 \pi+\varepsilon), \\
\psi(x)>0, \quad x \in(\varepsilon, 2 \pi-\varepsilon), \\
\operatorname{supp} \theta=[-\varepsilon, 2 \pi+\varepsilon], \\
\operatorname{supp} \psi=[\varepsilon, 2 \pi-\varepsilon], \quad 0<\varepsilon<\frac{1}{4} .
\end{gathered}
$$

Moreover, let $\widehat{\phi}_{1}(\xi)=\theta(\xi), \widehat{\phi}_{2}(\xi)=\psi(\xi), \xi \in \mathbb{R}$, and $\Phi=$ $\left(\phi_{1}, \phi_{2}\right)^{T}$. Then $\operatorname{rank}[\widehat{\Phi}(\xi+2 j \pi)]_{j \in \mathbb{Z}}=1, \xi \in \mathbb{R}$.

Proof. Note that $\phi_{i} \in \mathcal{S}(\mathbb{R}) \subset W_{\mu}^{1}(\mathbb{R}), i=1,2$.

We have the following two cases.

$\left(1^{\circ}\right)$ If $\xi \in(-\varepsilon+2 \ell \pi, \varepsilon+2 \ell \pi), \ell \in \mathbb{Z}$, then matrix

$$
\begin{array}{r}
{[\widehat{\Phi}(\xi+2 j \pi)]_{j \in \mathbb{Z}}=\left[\begin{array}{llllll}
\cdots & 0 & a & b & 0 & \cdots \\
\cdots & 0 & 0 & 0 & 0 & \cdots
\end{array}\right],} \\
a, b>0
\end{array}
$$

has a constant rank equal to 1 .

$\left(2^{\circ}\right)$ For $\xi \in(\varepsilon+2 \ell \pi, 2 \pi-\varepsilon+2 \ell \pi), \ell \in \mathbb{Z}$, the rank of matrix

$$
[\widehat{\Phi}(\xi+2 j \pi)]_{j \in \mathbb{Z}}=\left[\begin{array}{lllll}
\cdots & 0 & c & 0 & \cdots \\
\cdots & 0 & d & 0 & \cdots
\end{array}\right],
$$

where $c, d$ are non-zero values, is equal to 1 . An equivalent matrix is obtained for $\xi=\varepsilon+2 \ell \pi$ and $\xi=-\varepsilon+2 \ell \pi$, so we conclude that $\operatorname{rank}[\widehat{\Phi}(\xi+2 j \pi)]_{j \in \mathbb{Z}}=1$, for $\xi \in[\varepsilon+2 \ell \pi, 2 \pi-$ $\varepsilon+2 \ell \pi], \ell \in \mathbb{Z}$.

Considering these two cases, the rank of matrix $[\widehat{\Phi}(\xi+$ $2 j \pi)]_{j \in \mathbb{Z}}$ is a constant function on $\mathbb{R}, \operatorname{rank}[\widehat{\Phi}(\xi+2 j \pi)]_{j \in \mathbb{Z}}=1$, $\xi \in \mathbb{R}$.

Using functions $\theta$ and $\psi$ from Lemma 10, in the next lemma we construct the $p$-frame with four functions.

Lemma 11. Let the functions $\theta$ and $\psi$ satisfy all the conditions of Lemma 10. Moreover, let

$$
\begin{array}{r}
\widehat{\phi}_{k}(\xi)=\theta(\xi+2 k \pi), \quad \widehat{\phi}_{k+2}(\xi)=\psi(\xi+2 k \pi), \\
k=0,1,
\end{array}
$$

and $\Phi=\left(\phi_{0}, \phi_{1}, \phi_{2}, \phi_{3}\right)^{T}$. Then $\operatorname{rank}[\widehat{\Phi}(\xi+2 j \pi)]_{j \in \mathbb{Z}}=2$, $\xi \in \mathbb{R}$.

Proof. The proof is similar to the proof of Lemma 10.

$\left(1^{\circ}\right)$ If $\xi \in(-\varepsilon+2 \ell \pi, \varepsilon+2 \ell \pi), \ell \in \mathbb{Z}$, then matrix

$$
[\widehat{\Phi}(\xi+2 j \pi)]_{j \in \mathbb{Z}}=\left[\begin{array}{ccccccc}
\cdots & 0 & 0 & a^{1} & b^{1} & 0 & \cdots \\
\cdots & 0 & a^{2} & b^{2} & 0 & 0 & \cdots \\
\cdots & 0 & 0 & 0 & 0 & 0 & \cdots \\
\cdots & 0 & 0 & 0 & 0 & 0 & \cdots
\end{array}\right],
$$

where $a^{i}, b^{i}>0, i=1,2$, has a constant rank equal to 2 . 
$\left(2^{\circ}\right)$ For $\xi \in[\varepsilon+2 \ell \pi, 2 \pi-\varepsilon+2 \ell \pi], \ell \in \mathbb{Z}$, we have

$$
\operatorname{rank}[\widehat{\Phi}(\xi+2 j \pi)]_{j \in \mathbb{Z}}=\left[\begin{array}{cccccc}
\cdots & 0 & 0 & c^{1} & 0 & \cdots \\
\cdots & 0 & 0 & d^{1} & 0 & \cdots \\
\cdots & 0 & c^{2} & 0 & 0 & \cdots \\
\cdots & 0 & d^{2} & 0 & 0 & \cdots
\end{array}\right]=2 \text {, }
$$

where $c^{i}, d^{i}>0, i=1,2$.

We conclude that the rank of matrix $[\widehat{\Phi}(\xi+2 j \pi)]_{j \in \mathbb{Z}}$ is a constant function on $\mathbb{R}$ equal to 2 .

Lemma 10 can be easily generalised for an even number of functions $\phi_{i}, i=0, \ldots, 2 r-1$, with compactly supported $\widehat{\phi}_{i}, i=0, \ldots, 2 r-1$. The proof of the next theorem is similar to the previous proofs.

Theorem 12. Let the functions $\theta$ and $\psi$ satisfy all the conditions of Lemma 10. Moreover, let

$$
\begin{gathered}
\widehat{\phi}_{k}(\xi)=\theta(\xi+2 k \pi), \quad \widehat{\phi}_{k+r}(\xi)=\psi(\xi+2 k \pi), \\
k=0, \ldots, r-1, r \in \mathbb{N}
\end{gathered}
$$

and $\Phi=\left(\phi_{0}, \phi_{1}, \ldots, \phi_{2 r-1}\right)^{T}$.

The following statements hold.

$\left(1^{\circ}\right) \operatorname{rank}[\widehat{\Phi}(\xi+2 j \pi)]_{j \in \mathbb{Z}}=r$ for all $\xi \in \mathbb{R}$.

$\left(2^{\circ}\right) V_{\mu}^{p}(\Phi)$ is closed in $L_{\mu}^{p}$ for any $p \in[1, \infty]$.

$\left(3^{\circ}\right)\left\{\phi_{i}(\cdot-j) \mid j \in \mathbb{Z}, 0 \leq i \leq 2 r-1\right\}$ is a $p$-frame for $V_{\mu}^{p}(\Phi)$ for any $p \in[1, \infty]$.

Now we consider three functions with compact supports.

Lemma 13. Let the function $\theta$ satisfies all the conditions of Lemma 10 , and let $\tau \in C_{0}^{\infty}(\mathbb{R})$ and $\omega \in C_{0}^{\infty}(\mathbb{R})$ be positive functions such that

$$
\begin{gathered}
\tau(x)>0, \quad x \in(\varepsilon, \pi-\varepsilon) \cup(\pi+\varepsilon, 2 \pi-\varepsilon), \\
\omega(x)>0, \quad x \in(-3 \pi-\varepsilon,-\pi+\varepsilon), \\
\operatorname{supp} \tau=[\varepsilon, \pi-\varepsilon] \cup[\pi+\varepsilon, 2 \pi-\varepsilon], \\
\operatorname{supp} \omega=[-3 \pi-\varepsilon,-\pi+\varepsilon], \quad 0<\varepsilon<\frac{1}{4} .
\end{gathered}
$$

Moreover, let $\widehat{\phi}_{1}(\xi)=\theta(\xi), \widehat{\phi}_{2}(\xi)=\tau(\xi), \widehat{\phi}_{3}(\xi)=\omega(\xi), \xi \in \mathbb{R}$, and $\Phi=\left(\phi_{1}, \phi_{2}, \phi_{3}\right)^{T}$. Then $\operatorname{rank}[\widehat{\Phi}(\xi+2 j \pi)]_{j \in \mathbb{Z}}=2, \xi \in \mathbb{R}$.

Proof. We have four different forms for matrix $[\widehat{\Phi}(\xi+2 j \pi)]_{j \in \mathbb{Z}}$ and in all we show that the rank of matrix is equal to 2 .

Now we will show all possible cases. Denote with $a^{i}, i=$ $1,2,3$, and $b^{i}, i=1,2$, some positive values.

$\left(1^{\circ}\right)$ Consider

$$
\begin{array}{r}
{[\widehat{\Phi}(\xi+2 j \pi)]_{j \in \mathbb{Z}}=\left[\begin{array}{ccccccc}
\cdots & 0 & a^{1} & b^{1} & 0 & 0 & \cdots \\
\cdots & 0 & 0 & 0 & a^{2} & 0 & \cdots \\
\cdots & 0 & 0 & 0 & 0 & 0 & \cdots
\end{array}\right],} \\
\xi \in(-\varepsilon+2 \ell \pi, \varepsilon+2 \ell \pi) .
\end{array}
$$

$\left(2^{\circ}\right)$ Consider

$$
\begin{array}{r}
{[\widehat{\Phi}(\xi+2 j \pi)]_{j \in \mathbb{Z}}=\left[\begin{array}{cccccc}
\cdots & 0 & b^{1} & 0 & 0 & \cdots \\
\cdots & 0 & 0 & a^{2} & 0 & \cdots \\
\cdots & 0 & a^{3} & 0 & 0 & \cdots
\end{array}\right],} \\
\xi \in[\varepsilon+2 \ell \pi, \pi-\varepsilon+2 \ell \pi] .
\end{array}
$$

$\left(3^{\circ}\right)$ Consider

$$
\begin{aligned}
{[\widehat{\Phi}(\xi+2 j \pi)]_{j \in \mathbb{Z}} } & =\left[\begin{array}{ccccccc}
\cdots & 0 & b^{1} & 0 & 0 & 0 & \cdots \\
\cdots & 0 & 0 & a^{2} & b^{2} & 0 & \cdots \\
\cdots & 0 & 0 & 0 & 0 & 0 & \cdots
\end{array}\right], \\
\xi & \in(\pi-\varepsilon+2 \ell \pi, \pi+\varepsilon+2 \ell \pi) .
\end{aligned}
$$

$\left(4^{\circ}\right)$ Consider

$$
\begin{array}{r}
{[\widehat{\Phi}(\xi+2 j \pi)]_{j \in \mathbb{Z}}=\left[\begin{array}{ccccccc}
\cdots & 0 & b^{1} & 0 & 0 & 0 & \cdots \\
\cdots & 0 & 0 & 0 & b^{2} & 0 & \cdots \\
\cdots & 0 & a^{3} & 0 & 0 & 0 & \cdots
\end{array}\right],} \\
\xi \in[\pi+\varepsilon+2 \ell \pi, 2 \pi-\varepsilon+2 \ell \pi] .
\end{array}
$$

Remark 14. In Lemma 13 the support of the function $\omega$ must have an empty intersection with the supports of $\theta$ and $\tau$. In the opposite case, that is, $\operatorname{supp} \theta \cap \operatorname{supp} \tau \cap \operatorname{supp} \omega \neq \emptyset$, the rank of the matrix $[\widehat{\Phi}(\xi+2 j \pi)]_{j \in \mathbb{Z}}$ is a non-constant function on $\mathbb{R}$.

Lemma 13 can be easily generalised for functions $\phi_{i}, i=$ $0, \ldots, 3 r-1$, with compactly supported $\widehat{\phi}_{i}, i=0, \ldots, 3 r-$ 1. The proof of the next theorem is similar to the previous proofs.

Theorem 15. Let the functions $\theta, \tau$, and $\omega$ satisfy all the conditions of Lemma 13. Moreover, let

$$
\begin{aligned}
\widehat{\phi}_{k}(\xi) & =\theta(\xi+2 k \pi), \\
\widehat{\phi}_{k+r}(\xi) & =\tau(\xi+2 k \pi), \\
\widehat{\phi}_{k+2 r}(\xi) & =\omega(\xi+2 k \pi),
\end{aligned}
$$

$k=0, \ldots, r-1, r \in \mathbb{N}$, and $\Phi=\left(\phi_{0}, \phi_{1}, \ldots, \phi_{3 r-1}\right)^{T}$.

The following statements hold.

$\left(1^{\circ}\right) \operatorname{rank}[\widehat{\Phi}(\xi+2 j \pi)]_{j \in \mathbb{Z}}=2 r$ for all $\xi \in \mathbb{R}$.

$\left(2^{\circ}\right) V_{\mu}^{p}(\Phi)$ is closed in $L_{\mu}^{p}$ for any $p \in[1, \infty]$.

$\left(3^{\circ}\right)\left\{\phi_{i}(\cdot-j) \mid j \in \mathbb{Z}, 0 \leq i \leq 3 r-1\right\}$ is a $p$-frame for $V_{\mu}^{p}(\Phi)$ for any $p \in[1, \infty]$.

\section{Construction of Frames of Functions with Finite Regularities and Compact Supports}

We will recall the well-known construction of the $B$-spline functions in order to justify the rank properties of the corresponding matrices. 
Let $H(x), x \in \mathbb{R}$, be the characteristic function of the semiaxis $x \geq 0$; that is, $H(x)=0$ if $x<0$ and $H(x)=1$ if $x \geq 0$ (Heaviside's function). We construct a sequence $\left\{\phi_{n}\right\}_{n \in \mathbb{N}}$ in the following way. Let $\phi_{1}(x):=(H(x)-H(x-a)) / a, a>0$, $\phi_{2}:=\phi_{1} * \phi_{1}, \phi_{3}:=\phi_{1} * \phi_{1} * \phi_{1}, \ldots$; that is,

$$
\phi_{n}:=\underbrace{\phi_{1} * \phi_{1} * \cdots * \phi_{1}}_{n-1 \text { times }}, \quad n \in \mathbb{N},
$$

where $*$ denotes the convolution of the functions.

We obtain

$$
\begin{aligned}
& \begin{aligned}
& \phi_{2}(x)=\frac{1}{a^{2}}( x H(x)-2(x-a) H(x-a) \\
&+(x-2 a) H(x-2 a)), \\
& \phi_{3}(x)=\frac{1}{2 ! a^{3}}( x^{2} H(x)-3(x-a)^{2} H(x-a)+3(x-2 a)^{2} \\
&\left.\times H(x-2 a)-(x-3 a)^{2} H(x-3 a)\right), \\
& \phi_{4}(x)=\frac{1}{3 ! a^{4}}\left(x^{3} H(x)-4(x-a)^{3} H(x-a)\right. \\
&+ 6(x-2 a)^{3} H(x-2 a)-4(x-3 a)^{3} \\
& \times\left.H(x-3 a)+(x-4 a)^{3} H(x-4 a)\right) .
\end{aligned}
\end{aligned}
$$

Continuing in this manner, for all $n \in \mathbb{N}$, we have

$$
\begin{aligned}
\phi_{n}(x)=\frac{1}{a^{n}(n-1) !} \\
\times\left(\left(\begin{array}{c}
n \\
0
\end{array}\right) x^{n-1} H(x)-\left(\begin{array}{c}
n \\
1
\end{array}\right)(x-a)^{n-1}\right. \\
\quad \times H(x-a)+\left(\begin{array}{c}
n \\
2
\end{array}\right)(x-2 a)^{n-1} \\
\quad \times H(x-2 a)-\left(\begin{array}{c}
n \\
3
\end{array}\right)(x-3 a)^{n-1} H(x-3 a) \\
\quad+\cdots+(-1)^{n-1}\left(\begin{array}{c}
n \\
n-1
\end{array}\right)(x-(n-1) a)^{n-1} \\
\quad \times H(x-(n-1) a)+(-1)^{n}\left(\begin{array}{l}
n \\
n
\end{array}\right) \\
\left.\quad \times(x-n a)^{n-1} H(x-n a)\right) .
\end{aligned}
$$

Calculating the Fourier transform of functions $\phi_{n}, n \in \mathbb{N}$, we get

$$
\begin{gathered}
\widehat{\phi}_{1}(\xi)=\frac{-i}{a} v \cdot p \cdot\left(\frac{1}{\xi}\right)\left(e^{i a \xi}-1\right), \\
\widehat{\phi}_{2}(\xi)=\frac{(-i)^{2}}{a^{2}} v \cdot p \cdot\left(\frac{1}{\xi^{2}}\right)\left(e^{i a \xi}-1\right)^{2}, \\
\widehat{\phi}_{3}(\xi)=\frac{(-i)^{3}}{a^{3}} v \cdot p \cdot\left(\frac{1}{\xi^{3}}\right)\left(e^{i a \xi}-1\right)^{3} .
\end{gathered}
$$

Continuing in this manner, we obtain $\widehat{\phi}_{n}(\xi)=(-i)^{n} / a^{n} v \cdot p$. $\left(1 / \xi^{n}\right)\left(e^{i a \xi}-1\right)^{n}, n \in \mathbb{N}$, where $v \cdot p \cdot$ denotes the principal value.

Let $\Phi=\left(\phi_{1}, \phi_{2}, \ldots, \phi_{r}\right)^{T}, r \in \mathbb{N}$. matrix $[\widehat{\Phi}(\xi+2 j \pi)]_{j \in \mathbb{Z}}$ has for all $\xi \in \mathbb{R}$ the same rank as matrix

$$
\begin{aligned}
& R(\xi) \\
& =\left[\begin{array}{ccccccc}
\cdots & \alpha_{-4 \pi} \beta_{-4 \pi} & \alpha_{-2 \pi} \beta_{-2 \pi} & \alpha_{0} \beta_{0} & \alpha_{2 \pi} \beta_{2 \pi} & \alpha_{4 \pi} \beta_{4 \pi} & \cdots \\
\cdots & \alpha_{-4 \pi}^{2} \beta_{-4 \pi}^{2} & \alpha_{-2 \pi}^{2} \beta_{-2 \pi}^{2} & \alpha_{0}^{2} \beta_{0}^{2} & \alpha_{2 \pi}^{2} \beta_{2 \pi}^{2} & \alpha_{4 \pi}^{2} \beta_{4 \pi}^{2} & \cdots \\
\cdots & \alpha_{-4 \pi}^{3} \beta_{-4 \pi}^{3} & \alpha_{-2 \pi}^{3} \beta_{-2 \pi}^{3} & \alpha_{0}^{3} \beta_{0}^{3} & \alpha_{2 \pi}^{3} \beta_{2 \pi}^{3} & \alpha_{4 \pi}^{3} \beta_{4 \pi}^{3} & \cdots \\
\cdots & \alpha_{-4 \pi}^{4} \beta_{-4 \pi}^{4} & \alpha_{-2 \pi}^{4} \beta_{-2 \pi}^{4} & \alpha_{0}^{4} \beta_{0}^{4} & \alpha_{2 \pi}^{4} \beta_{2 \pi}^{4} & \alpha_{4 \pi}^{4} \beta_{4 \pi}^{4} & \cdots \\
& \vdots & \vdots & \vdots & \vdots & \vdots & \\
\cdots & \alpha_{-4 \pi}^{r} \beta_{-4 \pi}^{r} & \alpha_{-2 \pi}^{r} \beta_{-2 \pi}^{r} & \alpha_{0}^{r} \beta_{0}^{r} & \alpha_{2 \pi}^{r} \beta_{2 \pi}^{r} & \alpha_{4 \pi}^{r} \beta_{4 \pi}^{r} & \cdots
\end{array}\right],
\end{aligned}
$$

where $\alpha_{k}^{m}=v \cdot p \cdot(1 /(\xi-k))^{m}$ and $\beta_{k}^{m}=\left(e^{i a(\xi-k)}-1\right)^{m}$. Since $\operatorname{rank} R(\xi)=r, \xi \in \mathbb{R}$, we have the next result.

Theorem 16. Let $\Phi=\left(\phi_{k}, \phi_{k+1}, \ldots, \phi_{k+(r-1)}\right)^{T}$, for $k \in \mathbb{Z}, r \in$ $\mathbb{N}$. Then $V_{\mu}^{p}(\Phi)$ is closed in $L_{\mu}^{p}$ for any $p \in[1, \infty]$ and $\left\{\phi_{k+s}(\cdot-\right.$ j) $\mid j \in \mathbb{Z}, 0 \leq s \leq r-1\}$ is a $p$-Riesz basis for $V_{\mu}^{p}(\Phi)$ for any $p \in[1, \infty]$.

Remark 17. Let $k$ be a positive integer. We refer to [23] for the $L^{p}$-approximation order $k$. Shift-invariant spaces generated by a finite number of compactly supported functions in $L^{p}(\mathbb{R}), 1 \leq p \leq \infty$, were studied in [23] by Jia, who gave a characterization of the approximation order providing such shift-invariant spaces. Theorem 3 in [23] shows that the shiftinvariant space generated with the family of splines, which we constructed in Section 5, provides $L^{p}$-approximation order $k$.

Remark 18. (1) We refer to $[3,20]$ for the $\gamma$-dense set $X=$ $\left\{x_{j} \mid j \in J\right\}$. Let $\phi_{k}(x)=\mathscr{F}^{-1}(\theta(\cdot-k \pi))(x), x \in \mathbb{R}$. Following the notation of [20], we put $\psi_{x_{j}}=\phi_{x_{j}}$, where $\left\{x_{j} \mid j \in J\right\}$ is $\gamma$-dense set determined by $f \in V^{2}(\phi)=$ $V^{2}\left(\mathscr{F}^{-1}(\theta)\right)$. Theorems 3.1,3.2, and 4.1 in [20] give conditions and explicit form of $C_{p}>0$ and $c_{p}>0$ such that inequality $c_{p}\|f\|_{L_{\mu}^{p}} \leq\left(\sum_{j \in J}\left|\left\langle f, \psi_{x_{j}}\right\rangle \mu\left(x_{j}\right)\right|^{p}\right)^{1 / p} \leq C_{p}\|f\|_{L_{\mu}^{p}}$ holds. This inequality guarantees the feasibility of a stable and continuous reconstruction algorithm in the signal spaces $V_{\mu}^{p}(\Phi)[20]$.

(2) Since the spectrum of the Gram matrix $[\widehat{\Phi}, \widehat{\Phi}](\xi)$, where $\Phi$ is defined in Theorem 16, is bounded and bounded away from zero (see [7]), it follows that the family $\{\Phi(\cdot-j) \mid$ $j \in \mathbb{Z}$ \} forms a $p$-Riesz basis for $V_{\mu}^{p}(\Phi)$.

(3) Frames of the above sections may be useful in applications since they satisfy assumptions of Theorems 3.1 and 3.2 in [4]. They show that error analysis for sampling and reconstruction can be tolerated or that the sampling and reconstruction problem in shift-invariant space is robust with respect to appropriate set of functions $\phi_{k_{1}}, \ldots, \phi_{k_{r}}$. 


\section{Acknowledgments}

The authors are indebted to the referee for the valuable suggestions, which have contributed to the improvement of the presentation of the paper. The authors were supported in part by the Serbian Ministry of Science and Technological Developments (Project no. 174024).

\section{References}

[1] A. Aldroubi, Q. Sun, and W.-S. Tang, " $p$-frames and shift invariant subspaces of $L^{p}$," The Journal of Fourier Analysis and Applications, vol. 7, no. 1, pp. 1-21, 2001.

[2] A. Aldroubi, Q. Sun, and W.-S. Tang, "Non-uniform sampling in multiply generated shift-invariant subspaces of $L^{p}\left(\mathbb{R}^{d}\right)$," in Wavelet Analysis and Applications (Guangzhou, 1999), vol. 25 of AMS/IP Stud. Adv. Math., pp. 1-8, American Mathematical Society, Providence, RI, USA, 2002.

[3] A. Aldroubi, "Non-uniform weighted average sampling and reconstruction in shift-invariant and wavelet spaces," Applied and Computational Harmonic Analysis, vol. 13, no. 2, pp. 151161, 2002.

[4] A. Aldroubi and I. Krishtal, "Robustness of sampling and reconstruction and Beurling-Landau-type theorems for shiftinvariant spaces," Applied and Computational Harmonic Analysis, vol. 20, no. 2, pp. 250-260, 2006.

[5] A. Aldroubi and M. Unser, "Sampling procedures in function spaces and asymptotic equivalence with Shannon's sampling theory," Numerical Functional Analysis and Optimization, vol. 15, no. 1-2, pp. 1-21, 1994.

[6] A. Aldroubi, A. Baskakov, and I. Krishtal, "Slanted matrices, Banach frames, and sampling," Journal of Functional Analysis, vol. 255, no. 7, pp. 1667-1691, 2008.

[7] C. de Boor, R. A. DeVore, and A. Ron, "The structure of finitely generated shift-invariant spaces in $L^{2}\left(\mathbb{R}^{d}\right)$," Journal of Functional Analysis, vol. 119, no. 1, pp. 37-78, 1994.

[8] R. A. DeVore, B. Jawerth, and B. J. Lucier, "Image compression through wavelet transform coding," IEEE Transactions on Information Theory, vol. 38, no. 2, pp. 719-746, 1992.

[9] H. G. Feichtinger, "Banach convolution algebras of Wiener type," in Functions, Series, Operators, Vol. I, II (Budapest, 1980), vol. 35 of Colloq. Math. Soc. János Bolyai, pp. 509-524, NorthHolland, Amsterdam, The Netherlands, 1983.

[10] H. G. Feichtinger and K. Gröchenig, "A unified approach to atomic decompositions via integrable group representations," in Function Spaces and Applications (Lund, 1986), vol. 1302 of Lecture Notes in Math., pp. 52-73, Springer, Berlin, Germany, 1988.

[11] H. G. Feichtinger and K. H. Gröchenig, "Banach spaces related to integrable group representations and their atomic decompositions. I," Journal of Functional Analysis, vol. 86, no. 2, pp. 307340, 1989.

[12] H. G. Feichtinger, "Generalized amalgams, with applications to Fourier transform," Canadian Journal of Mathematics, vol. 42, no. 3, pp. 395-409, 1990.

[13] H. G. Feichtinger and K. Gröchenig, "Iterative reconstruction of multivariate band-limited functions from irregular sampling values," SIAM Journal on Mathematical Analysis, vol. 23, no. 1, pp. 244-261, 1992.

[14] H. G. Feichtinger and K. Gröchenig, "Theory and practice of irregular sampling," in Wavelets: Mathematics and Applications,
J. J. Benedetto and W. Frazier, Eds., Stud. Adv. Math., pp. 305363, CRC, Boca Raton, Fla, USA, 1994.

[15] H. G. Feichtinger, K. Gröchenig, and T. Strohmer, "Efficient numerical methods in non-uniform sampling theory," Numerische Mathematik, vol. 69, no. 4, pp. 423-440, 1995.

[16] H. G. Feichtinger and T. Strohmer, Eds., Gabor Analysis and Algorithms, Applied and Numerical Harmonic Analysis, Birkhäuser, Boston, Mass, USA, 1998.

[17] K. Gröchenig, "Describing functions: atomic decompositions versus frames," Monatshefte für Mathematik, vol. 112, no. 1, pp. $1-42,1991$.

[18] R. Q. Jia and C. A. Micchelli, "On linear independence for integer translates of a finite number of functions," Proceedings of the Edinburgh Mathematical Society, vol. 36, no. 1, pp. 69-85, 1993.

[19] S. Pilipović and S. Simić, "Frames for weighted shift-invariant spaces," Mediterranean Journal of Mathematics, vol. 9, no. 4, pp. 897-912, 2012.

[20] J. Xian and S. Li, "Sampling set conditions in weighted multiply generated shift-invariant spaces and their applications," Applied and Computational Harmonic Analysis, vol. 23, no. 2, pp. 171$180,2007$.

[21] A. Aldroubi and K. Gröchenig, "Non-uniform sampling and reconstruction in shift-invariant spaces," SIAM Review, vol. 43, no. 4, pp. 585-620, 2001.

[22] S. Simić, "Fréchet frames for shift invariant weighted spaces," Novi Sad Journal of Mathematics, vol. 39, no. 2, pp. 119-128, 2009.

[23] R.-Q. Jia, "Shift-invariant spaces on the real line," Proceedings of the American Mathematical Society, vol. 125, no. 3, pp. 785-793, 1997. 


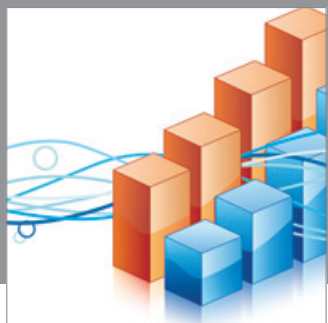

Advances in

Operations Research

mansans

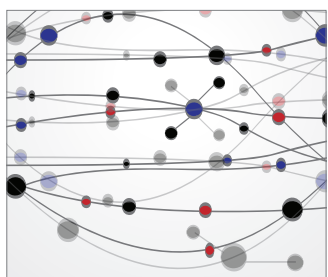

The Scientific World Journal
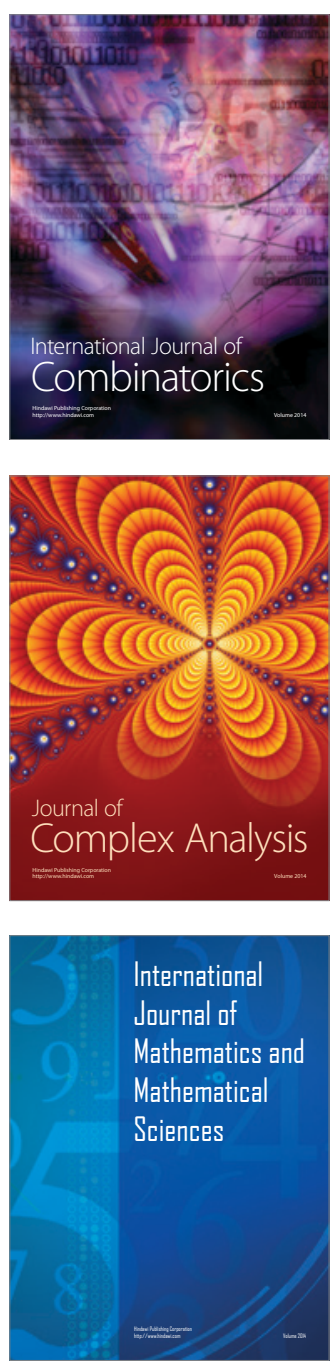
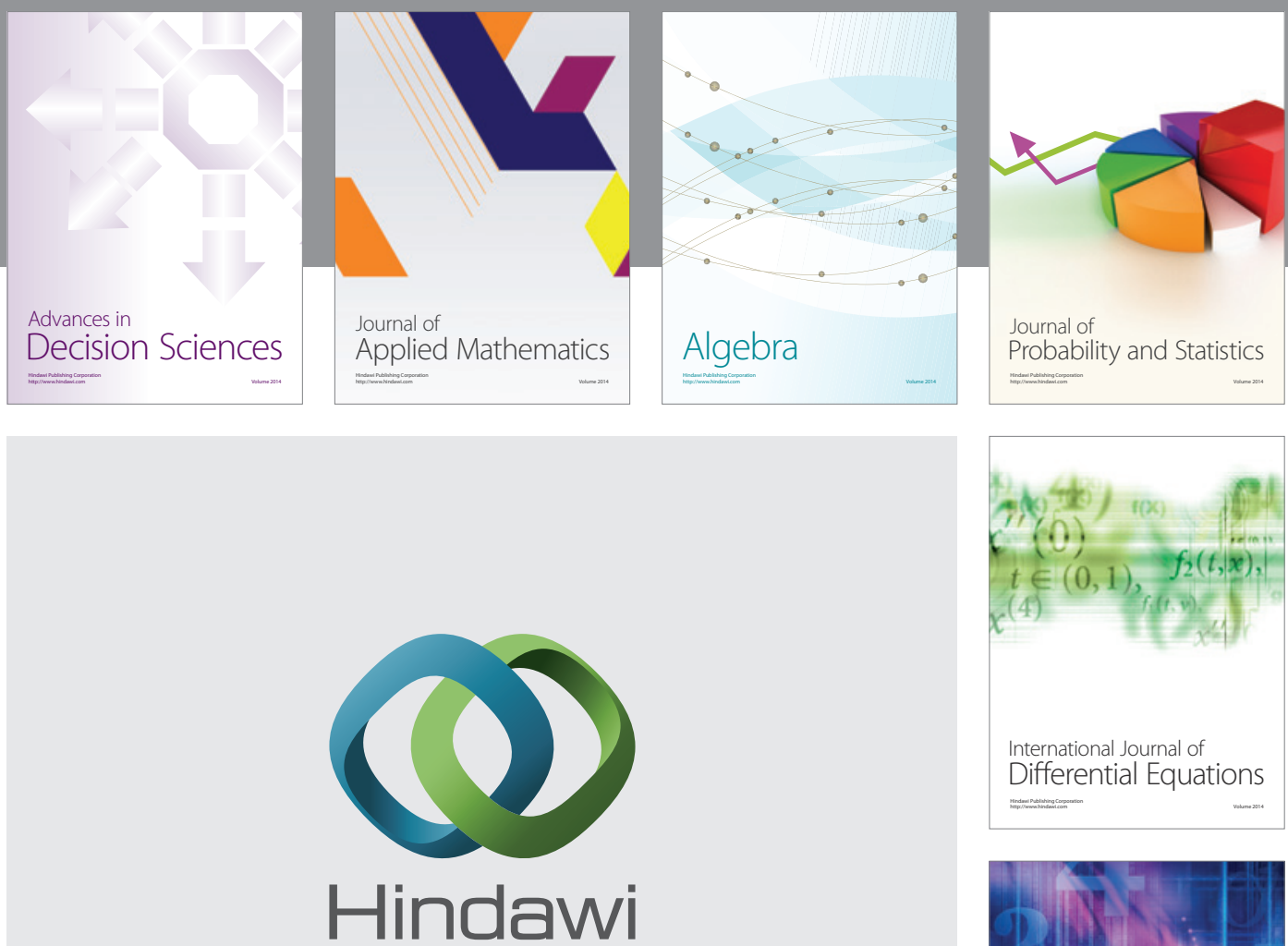

Submit your manuscripts at http://www.hindawi.com
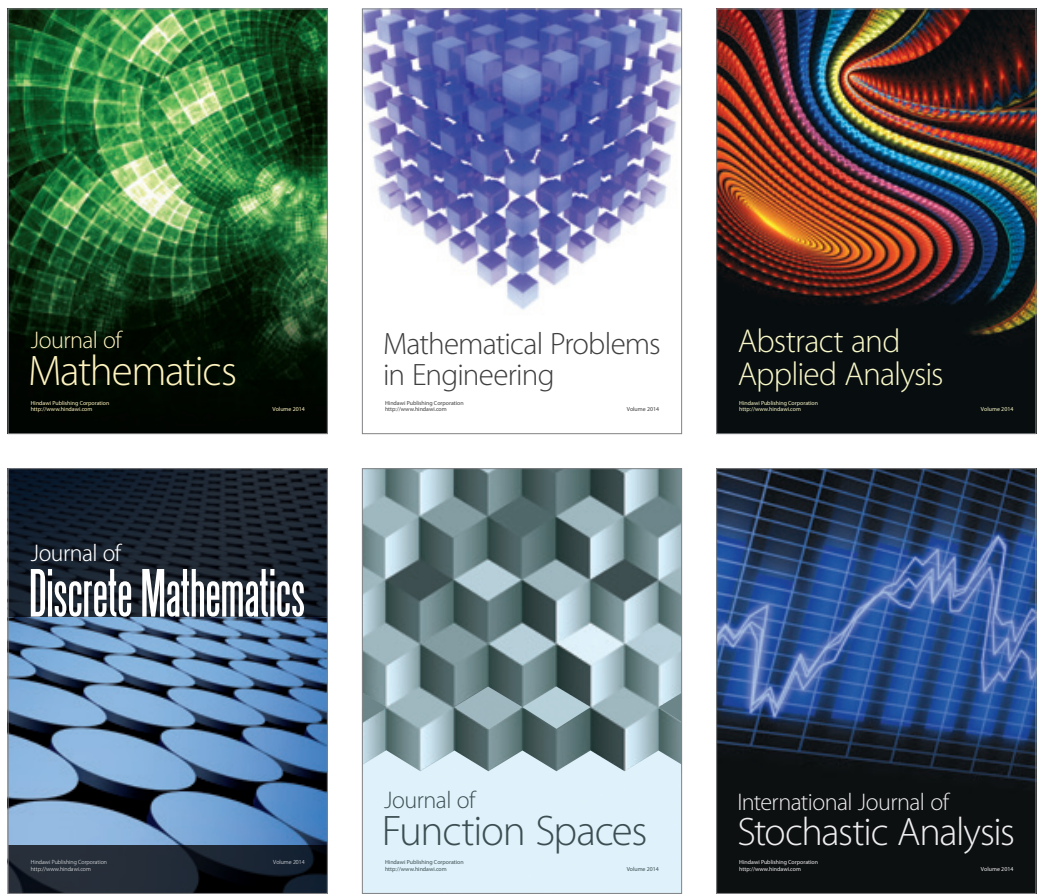

Journal of

Function Spaces

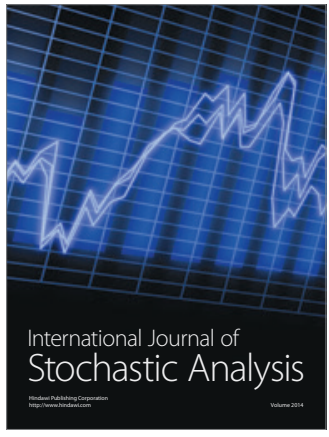

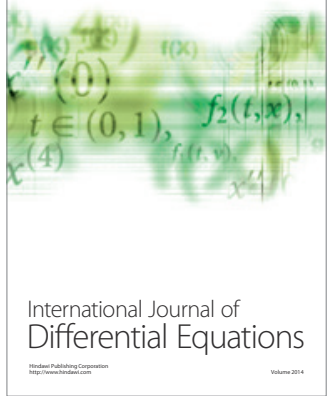
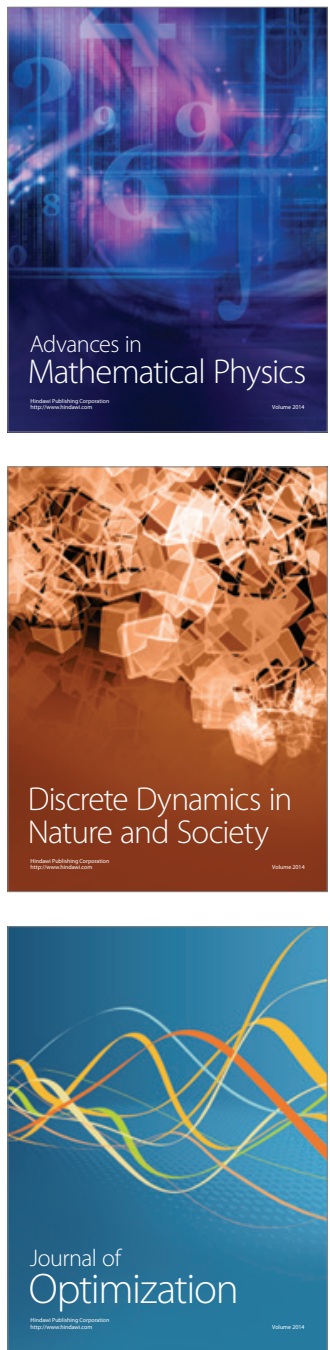